\title{
Endoscopic detection of ischaemia with a new probe indicates low oxygenation of gastric epithelium in portal hypertensive gastropathy
}

\author{
C Piasecki, J Chin, L Greenslade, N McIntyre, A K Burroughs, P A McCormick
}

\begin{abstract}
Changes in mucosal blood flow may be important in the pathogenesis of many conditions. Study of mucosal blood perfusion is difficult, and available methods have significant technical limitations. This study describes the development of an instrument for endoscopy, which indicates blood flow indirectly, by measuring the quantity of tissue oxygen that can diffuse from the mucosa to a luminal surface electrode. The instrument was used through an endoscope in patients with portal hypertension $(n=14)$, scleroderma $(n=3)$, disease controls $(n=7)$, and normal controls $(n=11)$. In portal hypertension readings were one quarter that in normal controls in both antrum (geometric mean (SEM) $35(1 \cdot 1))$, nanoamps $v 137(1 \cdot 1)$, and upper corpus $34(1 \cdot 1) v 125(1 \cdot 1))$. Scleroderma patients showed greatly reduced oxygen readings in both antrum (18 $(1 \cdot 2))$ and corpus $(24(1 \cdot 2))$, an expected but hitherto undiscovered result. These differences are highly significant $(p=0.0001)$, and the findings suggest that tissue hypoxia may contribute to mucosal changes in portal hypertensive gastropathy and in scleroderma.

(Gut 1995; 36: 654-656)
\end{abstract}

Keywords: endoscopy, ischaemia, portal hypertensive gastropathy.

Portal hypertensive gastropathy is a significant cause of bleeding from the stomach. The vascular mechanisms underlying the changes seen in this condition - that is, dilated vessels, ectasia, cherry red spots, etc - are uncertain. ${ }^{1}$ While there is agreement that the gastric mucosa is congested, though not inflamed, ${ }^{2}$ there is controversy as to whether perfusion is increased or decreased. ${ }^{3}$ Most reports suggest that gastric mucosal blood flow is increased in the presence of portal hypertension, both in experimental animals and in patients. ${ }^{3}$ Recently, however, two groups, using an identical laser Doppler method, reported diametrically opposite results in patients with portal hypertension, one finding an increase in mucosal blood flow the other a decrease. ${ }^{34}$ Whatever the cause of this discrepancy (selection of patients or difficulties inherent in the Doppler method), the situation remains unclear. The possibility that the circulation is decreased, is also supported by a study using hydrogen clearance. ${ }^{5}$ We set out to clarify this position, by measuring mucosal oxygenation using a new method. Our oxygen probe measures continuously the quantity of oxygen that can diffuse out of the epithelium to the instrument, and has been validated as far as is possible in animal studies. ${ }^{6-8}$ Thus the aims of this study were (a) to determine the feasibility of using this electrode endoscopically, and (b) to investigate possible changes in mucosal oxygen in some clinical states.

\section{Methods}

\section{Instrumentation}

The modification of the polarographic oxygen electrode to measure quantity of oxygen (not the pressure), has been fully described. ${ }^{6-8}$ Briefly, electrochemical reduction of oxygen by the instrument has been greatly increased by using a membrane highly permeable to oxygen and a large area. In animal studies the probe detected rapid changes from both serosal and mucosal surfaces induced by changing total blood flow, and serosal readings correlated well with total blood flow (measured by electromagnetic flowmetry). The probe was specially designed so that it would pass down the $3.7 \mathrm{~mm}$ diameter biopsy channel of a standard endoscope without danger of disrupting the probe, and to permit measurement under direct vision.

\section{Procedure}

After diagnostic endoscopy, the oxygen probe was passed down the biopsy channel and applied to the mucosa under direct vision. Undue pressure against mucosa was avoided by not allowing the probe to dimple the surface. Preliminary practice permitted the operator to ascertain how much pressure was required to lower the reading - that is, to obstruct the blood flow - and thereafter this effect could be avoided. Each application lasted 45 seconds, and was sufficient to obtain a steady reading. Usually two to three applications (but ranging from one to six ) were made at the same site, the sites being mid-antrum, upper corpus, and upper third of oesophagus. The last served as an in vivo control in tissue not permeable to oxygen. The endoscopist stated when he considered an application satisfactory and, to reduce bias, did not see the reading, while a second investigator observed the meter and stated when a steady reading was obtained. Because of time constraints and 


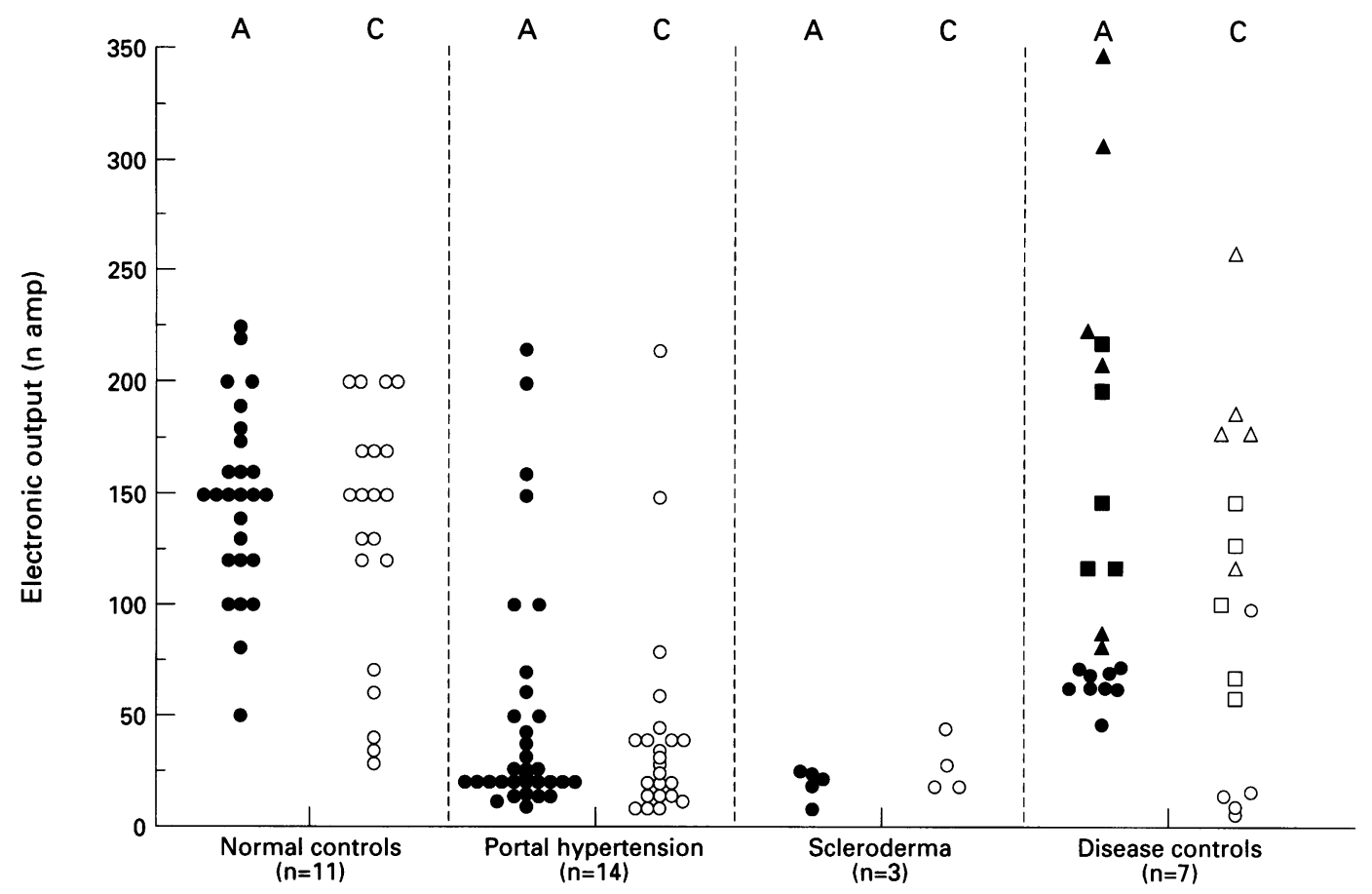

Amount of oxygenation of surface gastric epithelium in three groups of patients and a normal control group, as detected by the modified oxygen electrode during endoscopy. Each emblem represents a single reading at one point. $A=$ antrum, $C=u p p e r$ corpus. In the disease control group, circles=duodenal lesion, triangles =oesophagitis, squares=primary biliary cirrhosis without portal hypertension. For statistics, see results.

gastric motility, it was not possible to obtain readings at all three sites in every patient.

\section{Patients}

Permission for the procedure was obtained from the hospital ethics committee, and each patient gave written informed consent. Four groups of patients were studied. Eleven normal controls were designated as those who had no past history of upper gut disease, and the current endoscopy (performed for dyspepsia, melaena, or anaemia) was normal (aged 35-80, five male/six female). Fourteen patients had oesophageal varices, and 13 of these had erythematous or snake skin gastritis (aged 29-72, 11 male/three female). A third group comprised three patients (all female aged 45-66), with scleroderma; two had normal stomachs while the third had gastritis. In the fourth group, consisting of seven disease controls (aged 52-70, three male/four female), two had duodenal ulcer, four had oesophagitis, and one patient had primary biliary cirrhosis but without portal hypertension; the stomach was normal at gastroscopy. Blood haemoglobin concentrations were measured. All patients were given intravenous diazepam (Diazemuls 10-15 mg) and lignocaine throat spray before the procedure. Pethidine $(25 \mathrm{mg}$ intravenously) was given to four patients and hyoscine ( $25 \mathrm{mg}$ intravenously) to three. Two patients received both drugs. Patients were not receiving $\beta$ blockers, nitrites or other vasoactive drugs.

\section{Statistical methods}

Logarithms were used in analysis because of skewed distribution. Results are reported as geometrical mean (geometric SEM). To take account of the different number of readings in each area and each subject, mean values were estimated using the least squares method. Tests for differences between groups were carried out using analysis of variance and Turkey's test for pairwise differences between groups.

\section{Results}

The Figure shows the results. In 14 patients with portal hypertension, 13 of whom had gastropathy, gastric mucosal oxygen readings (in nanoamps) were greatly reduced, the geometric mean being roughly a quarter that in normal controls in both antrum (35 (1.1) $v$ $137(1 \cdot 1)$ respectively) and upper corpus (34 $(1 \cdot 1) v 125(1 \cdot 1))$. This is a highly significant difference $(p=0.0001)$. Thus most gastropathy patients showed values well below normal, this being especially evident in the antrum. All three scleroderma patients had very low readings in both antrum (18 (1.2) and corpus 24 $(1 \cdot 2))$. The disease control group showed a full spectrum but the lowest values were in two patients with streaking oesophagitis. Taking the groups together, all but one of the differences between the groups in the two sites were significant at the $5 \%$ value.

Haemoglobin concentrations (mean (SEM) were similar in all groups (normal controls $13 \cdot 1(0 \cdot 8) \mathrm{g} / \mathrm{dl}$, portal hypertensive gastropathy $12.6(1 \cdot 3)$, scleroderma $12.8(1 \cdot 8)$, disease controls $12.5(1 \cdot 9)$.

The mean coefficient of variation for antrum measurements in individual patients was: normal controls $4 \cdot 4 \%$, portal hypertensive $11.9 \%$, scleroderma $3.8 \%$, and disease controls $3.6 \%$. For corpus, these were $4.8 \%$, $10 \cdot 1 \%, 8 \cdot 7 \%$, and $14 \cdot 8 \%$ respectively (see discussion). In the study as a whole, differences 
between groups explained $56.7 \%$ and $36.4 \%$ of the total variation in antrum and corpus respectively; differences between subjects within groups explained a further $36 \cdot 6 \%$ and $56.3 \%$, therefore only $6.6 \%$ of the variation in antrum and $7 \cdot 3 \%$ of the variation in corpus measurements probably resulted from measurement differences within the same subjects.

\section{Discussion}

In this study we used a modified oxygen electrode to measure oxygen content during diagnostic endoscopy. This method proved to be reliable and robust under clinical conditions. The procedure added about five minutes to the endoscopy time.

We believe that the comparatively high coefficients of variation for repeated readings at same sites probably result from changes in blood flow because of discomfort, and not because of instrumentation. This is because in studies on anaesthetised animals, repeated applications had a 0 to $3 \%$ variability. ${ }^{68}$ Furthermore, of 86 pairs of readings at the same sites in the whole study, 15 were exactly the same and 18 were within $10 \%$ of each other. As the range of readings was from 15 to 350 , such high occurrence of same readings, goes against instrumental error and suggests variation caused by discomfort.

A number of techniques are available to assess mucosal perfusion in vivo. Laser Doppler, the most popular, relies on the number and velocity of particles flowing through vessels to depths of 1-2 mm. Hence it probably measures both mucosal and submucosal flow. Spectral analysis measures blood oxygenation but not flow nor delivery of oxygen to tissues. The hydrogen electrode does measure blood flow and gas delivery to tissue but this method is involved and laborious. In previous experiments in vivo, we showed that our flow sensitive oxygen probe measures both the amount of oxygen that diffuses through the epithelium to the instrument and, as the probe in continuously reducing this oxygen, it also measures the ability of the blood flow to replace tissue oxygen. ${ }^{6}$ It is thus a direct measure of oxygenation of surface cells, and indirectly a measure of blood flow.

We found that mucosal oxygen content was low in both patients with scleroderma and portal hypertension. The result would be expected in scleroderma but has not been previously described to our knowledge. The low level of mucosal oxygen in portal hypertensive gastropathy is more controversial. Most studies suggest there is increased gastric blood flow in this condition. ${ }^{3}$ However, two recent studies suggested it may be reduced, one using a hydrogen clearance, ${ }^{5}$ the other a laser
Doppler method. ${ }^{4}$ This discrepancy may result from the different methods used. Our method measures a new parameter, the quantity of oxygen diffusing to the electrode. Surmising the cause of our low results, they could be caused by low mucosal blood flow, shunting of mucosal flow, or to systemic hypoxaemia. Hypoxaemia is not infrequently seen in patients with decompensated cirrhosis and may result from a ventilation perfusion mismatch, a rightward shift in the oxyhaemoglobin dissociation curve related to increased 2,3-diphosphoglycerate in erythrocytes, failure of hypoxic pulmonary vasoconstriction or intrapulmonary shunts. Hypoxaemia is unlikely in our cases, however, as all were monitored by pulse oximetry during endoscopy. Neither was anaemia a factor as haemoglobin concentrations were similar in all patient groups. While this study does not throw light on the causes, it does show that surface epithelium is poorly oxygenated in portal hypertension. The explanation why laser Doppler measurements are generally high in this condition, would seem to be that the Doppler is measuring deep mucosal or submucosal shunt flow, whereas our oxygen probe and the hydrogen clearance give low results because they measure tissue diffusion of gases at surface epithelium.

In conclusion, we suggest that this endoscopic oxygen probe may be a useful tool in the investigation of mucosal oxygenation. Because it is based on a different physical principle it will probably complement rather than replace currently available techniques. Thus our findings together with other techniques, suggest that the mucosa is more susceptible to injury in portal hypertensive gastropathy, because surface epithelial oxygenation is low. This is perfectly compatible with increased total flow. Further studies are required to clarify this issue.

This work was presented at the United European Gastroenterology Week in Barcelona, 1993 (Gut 1993; 34 (suppl 3): S39.

1 D'Amico G, Monalbano L, Traina M, Pisa R, Menozzi M, Spano C, et al. Natural history of congestive gastropathy in cirrhosis. Gastroenterology 1990; 99: 1558-64.

2 Sarfeh IJ, Tarnawski A. Gastric mucosal vasculopathy in portal hypertension, Gastroenterology 1987; 93: 1129-31. 3 Panez J, Bordas JM, Pique JM, Bosch J, Garcia-Pagan JC, Feu $\mathrm{F}$, et al. Increased gastric mucosal perfusion in pastropathy. Gastroenterology 1992; 103: 1875-82.

4 Iwao $T$, Toyonaga $A$, Ikegami $M$, Oho $K$, Sumino $M$, Harada $\mathrm{H}$, et al. Reduced gastric mucosal blood flow in patients with portal-hypertensive gastropathy. Hepatology 1993; 18: 36-40

5 Nishiwaki H, Asai T, Sowa M, Umeyama K. Endoscopic measurement of gastric mucosal flow with special reference to the effect of sclerotherapy in patients with liver cirrhosis. Am $\mathcal{F}$ Gastroenterol 1990; 85: 34-7.

6 Piasecki C. A new method for the assessment of gut viability. Br F Surg 1981; 68: 319-22.

7 Piasecki C. First experimental results with the oxygen electrode as a local blood flow sensor in canine colon. Br $\mathrm{F}$ Surg 1985; 72: 452-3.

8 Piasecki C, Seifalian AM. Continuous intraoperative monitoring of hepatic blood perfusion using a noninvasive surface electrode. Dig Dis Sci 1990; 35: 399-405. 